Classification

Physics Abstracts

$61.50-63.10-64.70 \mathrm{~K}-63.90$

\title{
Dynamics of colliding molecules : a theory of coherent neutron scattering for the plastic phase of $\mathrm{CBr}_{4}$
}

\author{
M. Descamps \\ Equipe de Dynamique des Cristaux Moléculaires (*), Université de Lille I, 59655 Villeneuve d'Ascq Cedex, France
}

(Reçu le 9 mai 1983, révisé le 7 octobre, accepté le 15 novembre 1983)

\begin{abstract}
Résumé. - On étudie dans cet article le comportement dynamique coopératif de molécules soumises à des interactions d'origine stérique. A cet effet on étend le modèle de Ising cinétique pour décrire un cristal plastique. Une approximation de niveau champ moyen est proposée pour écrire les probabilités de transition d'un système à orientations multiples avec des incompatibilités stériques dans certaines configurations. On obtient ainsi la fonction de diffusion neutronique cohérente $S(Q, \omega)$ du $\mathrm{CBr}_{4}$ en phase plastique. Les corrélations invoquées induisent une forte dispersion des temps de relaxation $\tau(\mathbf{Q})$ qui explique le comportement expérimental.
\end{abstract}

\begin{abstract}
The cooperative dynamical behaviour of disordered rotating molecules subject to steric hindrance is studied by extending Glauber's kinetic Ising model. A mean-field level approximation of the transition probabilities is proposed which describes multiorientational systems with steric hindrance. The coherent neutron scattering function $S(\mathbf{Q}, \omega)$ of $\mathrm{CBr}_{4}$ in its plastic phase is obtained. The invoked correlations induce a strong $\mathbf{Q}$ dispersion of the HWHM $\Gamma(\mathbf{Q})$ which explains the experimental behaviour.
\end{abstract}

\section{Introduction.}

In some molecular crystals showing orientational disorder (plastic crystals), the molecular packing is so high that the steric interactions play a very important rôle. It has been shown that they strongly contribute to a number of static properties such as $P_{1}(\Omega)$ the time averaged orientational probability as well as the joint probability $P_{2}\left(\Omega_{i} \Omega_{j}\right)$ which expresses the presence of intermolecular correlations.

Several statistical approaches to this problem have been previously published [1-3]. In the particular case of $\mathrm{CBr}_{4}$, they enabled us to give a microscopic interpretation of the static coherent neutron scattering $S(\mathbf{Q})$ which shows a very intense and anisotropic behaviour [2].

Concerning critical scattering which is sometimes observed near the order disorder phase transitions - as is the case for $\mathrm{CD}_{4}$ [4] - the energies at work are of the order of $k T_{\mathrm{c}} / Z(Z=$ coordination number $)$, and mean-field expressions for the dynamic suscepti-

$\left(^{*}\right)$ ERA du CNRS 465. bility can be derived in various ways giving rise to an interpretation of the observed critical slowing down of the orientational fluctuations. These theories cannot be immediately extended to the case of the energies of a higher level implicated in steric effects. We have recently proposed a mean-field level approximation for writing a compatibility function suitable to describe this type of interactions statistically [5].

In the present paper we use it to give a dynamic description of a molecular system subjected to strong reorientational hindrance. We build up a model by which the system is presumed to follow stochastic processes. The time evolution of this system is described by a Markoffian master equation which is an extension of the kinetic Ising model first introduced by Glauber [6-8].

In $\S 1$, we propose forms of the transition probabilities which accurately describe multiorientational systems with steric hindrance. In $\S 2.3$ we consider the particular case of $\mathrm{CBr}_{4}$ in order to obtain the expression for the coherent neutron scattering function $S(\mathbf{Q}, \omega)$ whose $\mathbf{Q}$-space variations have been measured experimentally elsewhere $[9,10,13]$. 


\section{The master equation.}

With a view to studying coherent neutron scattering we consider the $D$ sterically different orientations that can be occupied by each molecule. We characterize these $D$ possible configurations by the following function :

$$
\begin{aligned}
\mu_{l}^{i}\left(S_{i}\right) & =1 \text { if the orientation } S_{i} \text { of the } i \text { th molecule is effectively } l(l \in(1, D)) \\
& =0 \text { otherwise }
\end{aligned}
$$

the dynamical description of the system of $N$ molecules requires the knowledge of the probability distribution $P\left(S_{1}, \ldots, S_{N} ; t\right)$ of the molecular system in the state $\left\{S_{1}, \ldots, S_{N}\right\}=\{S\}$ at time $t$. The time dependence of $P(\{S\} ; t)$ is assumed to be governed by a Markoffian master equation

$$
\begin{aligned}
\frac{\mathrm{d}}{\mathrm{d} t} P\left(S_{1}, \ldots,\left(S_{j}=l\right), \ldots S_{N} ; t\right)= & -\sum_{j=1}^{N} \sum_{\substack{k=1 \\
\neq l}}^{D} \omega_{j}(l \rightarrow k) P\left(S_{1}, \ldots,\left(S_{j}=l\right), \ldots S_{N} ; t\right) \\
& +\sum_{j=1}^{N} \sum_{\substack{k=1 \\
\neq l}}^{D} \omega_{j}(k \rightarrow l) P\left(S_{1}, \ldots\left(S_{j}=k\right), \ldots S_{N} ; t\right)
\end{aligned}
$$

where $\omega_{j}(l \rightarrow k)$ denotes the transition probability of the $j$ th molecule going from state $l$ to state $k$. The second summation runs over the orientations $k$ which can be reached from $l$. The first term on the right hand side is the loss by flipping out and the second term is the gain. In the present description the heat bath is not treated explicitly : it gives rise to spontaneous flips, the probability of which depends of the equilibrium configurations of the neighbouring molecules. The transition probabilities $\omega_{j}$ must have a form which satisfy detailed balance in equilibrium. Consequently :

$$
\frac{\omega_{j}(l \rightarrow k)}{\omega_{j}(k \rightarrow l)}=\frac{P_{0}\left(S_{1}, \ldots\left(S_{j}=k\right), \ldots, S_{N}\right)}{P_{0}\left(S_{1}, \ldots\left(S_{j}=l\right), \ldots, S_{N}\right)}
$$

Concerning the all or nothing interactions that are the steric repulsions, the equilibrium probability is proportional to the product of compatibility functions $A\left(S_{j} S_{i}\right)$ :

$$
P_{0}\left(S_{1}, \ldots, S_{N}\right) \propto \prod_{\langle j i\rangle} A_{j i}\left(S_{j} S_{i}\right) \prod_{i} \prod_{l}\left(b_{l}^{i}\right)^{\mu_{i}^{i}\left(S_{i}\right)}
$$

The product, runs over all possible pairs $\langle j i\rangle$ and

$$
\begin{aligned}
& A_{j i}\left(S_{i} S_{j}\right)=1 \text { if the configurations } S_{i} \text { and } S_{j} \text { of neighbouring molecules } \\
& \text { are sterically compatible } \\
& =0 \text { otherwise }
\end{aligned}
$$

$b_{l}^{i}$ is a possible activity of the orientation $l$ at the site $i$. This term is specially useful to express the effect of an external perturbation. Thus (3) reduces to

$$
\frac{\omega_{j}(l \rightarrow k)}{\omega_{j}(k \rightarrow l)}=\frac{\prod_{\langle j i\rangle}^{n n} A_{j i}\left(k, S_{i}\right) b_{k}^{j}}{\prod_{\langle j i\rangle}^{n n} A_{j i}\left(l, S_{i}\right) b_{l}^{j}}
$$

in which the products run over the sites sterically connected with $j$. Most generally the compatibility function can be written as follows [11]

$$
A_{j i}\left(S_{j}, S_{i}\right)=1-C_{j i}\left(S_{j}\right) C_{i j}\left(S_{i}\right)
$$

where $C_{i j}\left(S_{i}\right)=1$ if the molecule in the state $S_{i}$ sterically occupies the $i j$ edge $=0$ otherwise.

We have shown in a previous paper (5) that a mean-field level approximation for the steric effects is obtained by taking the average of $A_{j i}\left(S_{j}, S_{i}\right)$ in the following approximate way :

$$
\left\langle A_{j i}\left(S_{j} S_{i}\right)\right\rangle_{\langle i\rangle}=1-C_{j i}\left(S_{j}\right)\left\langle C_{i j}\left(S_{i}\right)\right\rangle_{\langle i\rangle}
$$


(where $\left\langle C_{i j}\left(S_{i}\right)\right\rangle_{\langle i\rangle}$ is the averaged sterical occupation rate of the $i j$ edge by the $i$ th molecule) so that, in this approximation, the numerator and denominator of (6) are only functions of the state in $j$ when the transition has occurred. Equation (6) determines the transition probabilities up to an arbitrary factor and we may write :

$$
\omega_{j}(l \rightarrow k)=\frac{1}{\alpha_{l k}} \prod_{\langle j i\rangle}^{n n}\left\langle A_{j i}\left(k, S_{i}\right)\right\rangle_{\langle i\rangle}
$$

$\alpha_{l k}$ is a temperature dependent factor which can be considered as a measure of the time scale on which the reorientations take place. It is a non collective parameter given by the height of the potential barrier dividing the $l$ and $k$ orientations. It will be specified in the next section. The correlation of the molecular motion occurs because of the fact that the reorientation from $l$ to $k$ is possible only if $k$ is a state sterically compatible with the states of the neighbours (Fig. 1).

In order to study dynamical responses it is more convenient to study the time behaviour of the expectation values of $\mu_{m}^{i}$.

$$
\left\langle\mu_{m}^{i}(t)\right\rangle=\sum_{\{S\}} \mu_{m}^{i} P(\{S\} ; t) .
$$

We multiply both sides of (2) by $\mu_{m}^{i}$ and sum over $\{S\}$. Separating terms with $j=i$ from those with $j \neq i$ it is straightforward to see that only the $j=i$ term occurs. Since the mean-field expression for $\omega_{i}(9)$ is only a function of $S_{i}$ this last term reads :

$$
\begin{aligned}
& -\sum_{S_{i}} \sum_{k \neq l} \mu_{m}^{i} \omega_{i}(l \rightarrow k) \sum_{\{S\} \neq S_{i}} P\left(S_{1}, \ldots,\left(S_{i}=l\right), \ldots S_{N} ; t\right) \\
& +\sum_{S_{i}} \sum_{k \neq l} \mu_{m}^{i} \omega_{i}(k \rightarrow l) \sum_{\{S\} \neq S_{i}} P\left(S_{1}, \ldots,\left(S_{i}=k\right), \ldots S_{N} ; t\right) .
\end{aligned}
$$

Due to the definition (1) of $\mu^{i}$ and since the probability of orientation $m$ at site $i$ reads :

$$
\left\langle\mu_{m}^{i}\right\rangle=\sum_{\{S\} \neq S_{i}} P\left(S_{1}, \ldots,\left(S_{i}=m\right), \ldots, S_{N} ; t\right)
$$

the dynamics of the crystal are described by the following system of $D$ equations :

$$
\frac{\mathrm{d}}{\mathrm{d} t}\left\langle\mu_{m}^{i}(t)\right\rangle=-\left\langle\mu_{m}^{i}(t)\right\rangle \sum_{k \neq m}^{D} \omega_{i}(m \rightarrow k)+\sum_{k \neq m}^{D}\left\langle\mu_{k}^{i}(t)\right\rangle \omega_{i}(k \rightarrow m) .
$$

Owing to (9) and (8) and the definition of the $C_{i j}$, the $\omega_{i}$ terms can be expressed in terms of expectation values of $\mu_{n}^{j}$ in the neighbouring sites of $i$; a detailed expression will be given in $\S 2$.

\section{Intermolecular correlations and dynamics of $\mathrm{CBr}_{4}$.}

2.1 THE MODEL. - The structure refinement [12] of the plastic phase of $\mathrm{CBr}_{4}$ shows that the disordered bromide atoms of a molecule are preferentially located in the vicinity of the twofold axes and, on one site, this location can be expressed by the use of a model of six distinguishable discrete orientations with local symmetry $\overline{4} 2 \mathrm{~m}$ (Fig. 2) [10].

Because of the steric repulsion, two nearest-neighbour molecules cannot simultaneously bring a bromide atom into the vicinity of the [110] axis which joins the two molecules so that, for example, the situations shown in figure 4 are impossible.
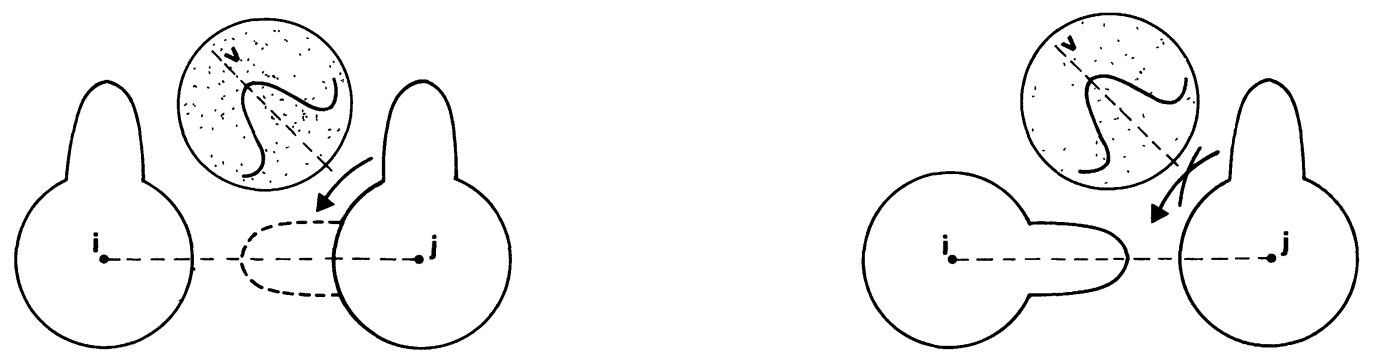

Fig. 1. - 1a allowed transition; $1 \mathrm{~b}$ forbidden transition due to the steric occupation of the $i-j$ edge by the molecule $i$. 


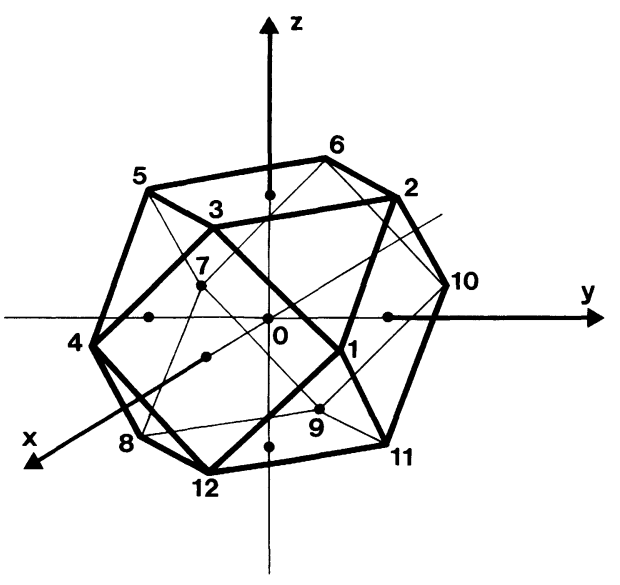

a)

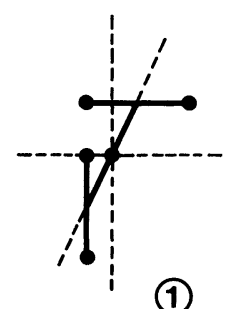

(1)

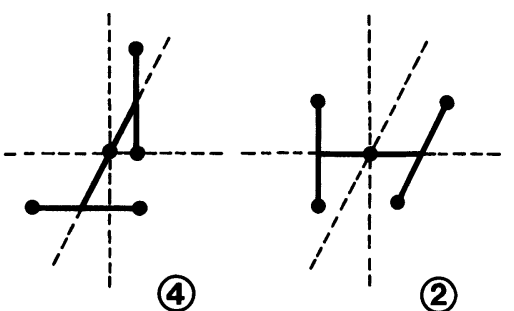

(2)

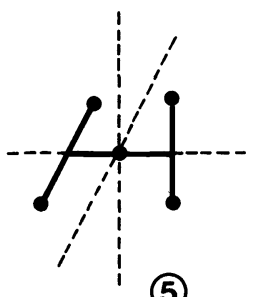

(5)
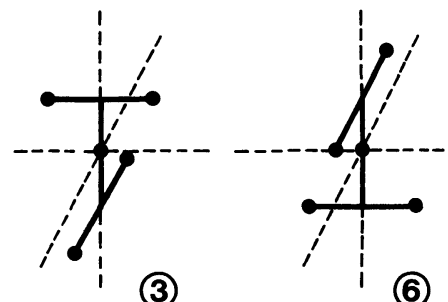

b)

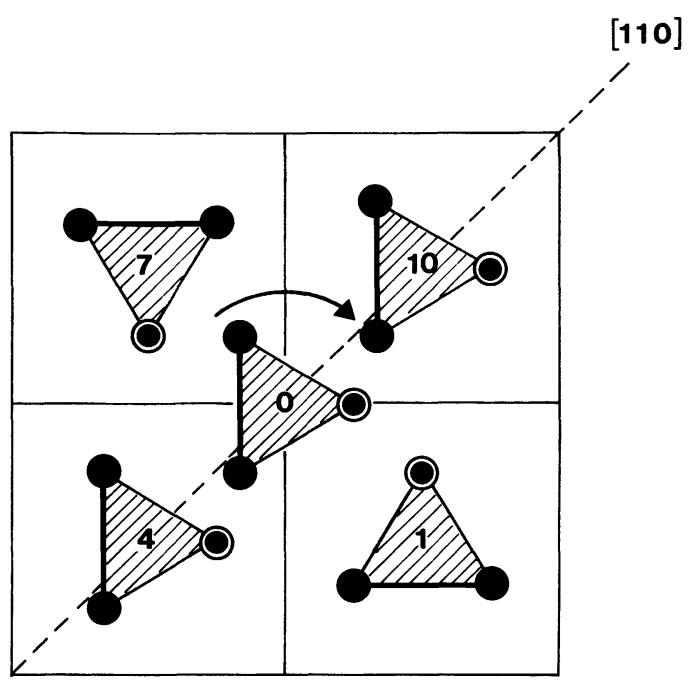

Fig. 3. - Steric effect between $\mathrm{CBr}_{4}$ molecules. The hatched triangles are the projections of $\mathrm{CBr}_{4}$ tetrahedra on the 001 plane. The rotation shown is impossible due to the resulting steric contact between the 0 and 10 molecules.

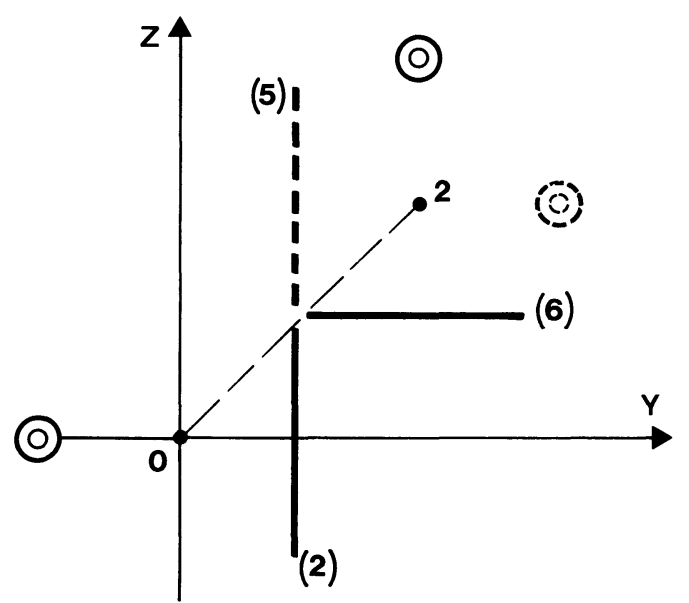

Fig. 4. - Example of impossible configurations in the (100) plane.
Fig. 2. - 2a numbering of the sites near the origin;

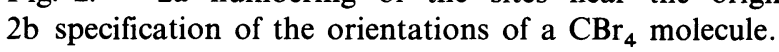

In the dynamic study of the model, the corresponding collective behaviour is taken into account in the way shown in figure 3 . As we have pointed out in $\S 1$ we only consider transition probabilities which connect states where the position of one single tetrahedron is changed while all the others remain unchanged.

Due to the symmetry, starting from a given orientation one can reach four different orientations by rotating around one of the fourfold or one of the threefold axes. The fifth orientation cannot be reached by a jump equivalent to the preceding ones. So that we should a priori introduce two different relaxation times which are the following if we take for example, the orientation 1 as the starting one

$$
\begin{aligned}
& \alpha_{12}=\alpha_{15}=\alpha_{13}=\alpha_{16}=\tau_{1} \\
& \alpha_{14}=\tau_{2} .
\end{aligned}
$$

To simplify the formulation we shall assume that $\tau_{1}=\tau_{2}=\tau$. So that for a molecule 0 surrounded by its twelve neighbours we can write (12) as the following system of equations $(m=1,6)$.

$$
\tau \frac{\mathrm{d}\left\langle\mu_{m}^{0}\right\rangle}{\mathrm{d} t}=-\left\langle\mu_{m}^{0}\right\rangle\left\{\sum_{k \neq m}^{6} \prod_{\langle 0 j\rangle}^{12}\left\langle A_{0 j}\left(k, S_{j}\right)\right\rangle_{\langle j\rangle} b_{k}^{0}\right\}+\left\{\left[\sum_{k \neq m}^{6}\left\langle\mu_{k}^{0}\right\rangle\right] \prod_{\langle 0 j\rangle}^{12}\left\langle A_{0 j}\left(m, S_{j}\right)\right\rangle_{\langle j\rangle} b_{m}^{0}\right\} .
$$


The precise expression for the compatibility function in the mean-field approximation is

$$
\begin{aligned}
\left\langle A_{0 j}\left(k, S_{j}\right)\right\rangle_{\langle j\rangle} & =1 \text { if the central } 0 \text { molecule in the } k \text { state does not sterically occupy the } 0 j \text { edge } \\
& =1-\left\langle C_{j 0}\right\rangle_{\langle j\rangle} \text { otherwise. }
\end{aligned}
$$

In the present case, the edge 02 for example, can be sterically occupied by the molecule 2 in the states 5 and 6 (Fig. 4) and

$$
\left\langle C_{20}\right\rangle_{\langle 2\rangle}=\left\langle\mu_{5}^{2}\right\rangle+\left\langle\mu_{6}^{2}\right\rangle .
$$

If the orientation in 0 is 2 :

$$
\left\langle A_{02}\left(2, S_{2}\right)\right\rangle_{\left\langle S_{2}\right\rangle}=1-\left\langle\mu_{5}^{2}\right\rangle-\left\langle\mu_{6}^{2}\right\rangle
$$

and the global contribution of $k=2$ to equation (13) is :

$$
\begin{aligned}
\prod_{\langle 0 j\rangle}^{12}\left\langle A_{0 j}\left(2, S_{j}\right)\right\rangle_{\langle j\rangle}=\left(1-\left\langle\mu_{5}^{2}\right\rangle-\left\langle\mu_{6}^{2}\right\rangle\right)\left(1-\left\langle\mu_{5}^{11}\right\rangle-\left\langle\mu_{3}^{11}\right\rangle\right) \times & \\
& \times\left(1-\left\langle\mu_{5}^{4}\right\rangle-\left\langle\mu_{1}^{4}\right\rangle\right)\left(1-\left\langle\mu_{5}^{7}\right\rangle-\left\langle\mu_{4}^{7}\right\rangle\right)
\end{aligned}
$$

owing to the fact that only the molecules situated on the sites $2,11,4$ and 7 may have steric contacts with the central molecule in the orientation 2.

The other equations of the system (13) can be deduced from (15) by symmetry analysis. We thus get homogeneous system of differential equations linking the time evolution of one molecule to the time evolution of its neighbours.

In our interaction model, we made two restrictive assumptions :

- discrete nature of the molecular orientations,

- strict steric hindrance for some molecular configurations.

In reality these hypotheses are slightly relaxed. Without studying completely the intermolecular potentials which would give rise to a very complicated model, we can introduce the softening of the intermolecular repulsion through one single parameter $\lambda$ occurring in the compatibility function as follows :

$$
A\left(S_{i} S_{j}\right)=1-\lambda C_{i j}\left(S_{i}\right) C_{j i}\left(S_{j}\right) \quad(0<\lambda<1)
$$

so that

$$
\begin{aligned}
A\left(S_{i} S_{j}\right) & =1-\lambda=\mathrm{e}^{-\beta W} \text { if } S_{i} \text { and } S_{j} \text { sterically interfere } \\
& =1 \text { otherwise }
\end{aligned}
$$

- $W$ is the mean repulsive energy of neighbouring molecules in unfavourable relative configurations.

2.2 The SUSCEPTIBILITY MATRIX. - The linear response to a slightly time dependent local perturbation is easily derived from equations (13) and (15) by linearization

- at equilibrium $b_{m}^{0}=1$ and $\left\langle\mu_{l}^{0}\right\rangle=1 / 6(\forall m, l)$,

- under the perturbation $b_{m}^{0}=1+\beta_{m}^{0}$ and $\left\langle\mu_{l}^{0}\right\rangle=\frac{1}{6}+\delta_{l}^{0}$.

For the orientation 1 the equation reads :

$$
\begin{aligned}
\left(\frac{3}{2}\right)^{4} \tau \frac{\mathrm{d} \delta_{1}^{0}}{\mathrm{~d} t}= & -6 \delta_{1}^{0}+\beta_{1}^{0}-\frac{1}{6} \sum_{m=1}^{6} \beta_{m}^{0}-\frac{3}{2}\left(\delta_{3}^{3}+\delta_{4}^{3}+\delta_{4}^{7}+\delta_{5}^{7}+\delta_{2}^{10}+\delta_{4}^{10}+\delta_{4}^{12}+\delta_{6}^{12}\right)+ \\
& +\frac{1}{2}\left(\delta_{1}^{1}+\delta_{2}^{1}+\delta_{5}^{2}+\delta_{6}^{2}+\delta_{3}^{3}+\delta_{4}^{3}+\delta_{1}^{4}+\delta_{5}^{4}+\delta_{2}^{5}+\delta_{6}^{5}+\delta_{1}^{6}+\delta_{3}^{6}+\delta_{4}^{7}+\delta_{5}^{7}+\delta_{2}^{8}+\delta_{3}^{8}+\right. \\
& \left.+\delta_{1}^{9}+\delta_{6}^{9}+\delta_{2}^{10}+\delta_{4}^{10}+\delta_{3}^{11}+\delta_{5}^{11}+\delta_{4}^{12}+\delta_{6}^{12}\right)
\end{aligned}
$$

the feature of which is that only the orientations 3 and 4 on the site 3,4 and 5 on the site 7,2 and 4 on the site 10 and 4 and 6 on the site 12 are sterically involved with the central site in the 1 orientation.

The six coupled equations of type (19) can be solved by Fourier transform, giving rise to a system of equations for $\delta_{m}(q, t)$ and $\beta_{l}(q)$, the Fourier transforms of the fluctuations of the orientational probabilities and of the activities respectively.

If the perturbations vary harmonically in time, $\beta_{m}^{0}(t)=\beta_{m}^{0} \mathrm{e}^{i \omega t}$ one can define a susceptibility matrix by the relation

$$
\delta_{m}(\mathbf{q}, t)=\sum_{l} \chi_{m l}(\mathbf{q}, \omega) \beta_{l}(\mathbf{q}) \mathrm{e}^{-i \omega t}
$$


According to the general fluctuation dissipation theorem [14] it can be expressed in terms of equilibrium correlation functions

$$
\begin{aligned}
\chi_{m l}(\mathbf{q}, \omega) & =\chi_{m l}(\mathbf{q}, 0)-i \omega \int_{0}^{\infty} \sum_{\mathbf{r}}\left\langle\delta_{m}^{0}(0) \delta_{l}^{r}(t)\right\rangle_{\mathrm{eq}} \mathrm{e}^{i \mathbf{q r}} \mathrm{e}^{-i \omega t} \mathrm{~d} t \\
& =\chi_{m l}(\mathbf{q}, 0)-i \omega \int_{0}^{\infty} \Phi_{m l}(q, t) \mathrm{e}^{-i \omega t} \mathrm{~d} t
\end{aligned}
$$

$\Phi_{l m}(q, t)$ is the relaxation function $\left(\Phi_{m l}(\mathbf{q}, t)=\Phi_{l m}(-\mathbf{q},-t)\right)$.

As a particular point :

$$
\chi_{m l}(\mathbf{q}, \omega=0)=\sum_{\mathbf{r}}\left\langle\delta_{m}^{0} \delta_{l}^{r}\right\rangle_{\text {eq }} \mathrm{e}^{i q r}
$$

which is the static susceptibility $\chi_{l m}(\mathbf{q})$.

Use being made of the evident relation :

$$
\sum_{m=1}^{6} \delta_{m}(\mathbf{q}, t)=0
$$

which completes the system of the Fourier transforms of equations (17), we obtain $\chi(q, \omega)$ (given in the appendix).

2.3 THE NEUTRON SCATTERING CROSS SECTION. The coherent cross section is proportional to the coherent scattering function [15-17]

$$
\begin{array}{r}
I_{\mathbf{c}}(\mathbf{Q}, \omega)=\sum_{\mathbf{r}} \int_{-\infty}^{+\infty} \sum_{m} \sum_{l} f_{m}^{0} f_{l}^{r^{*}}\left\langle\delta_{m}^{0}(0) \delta_{l}^{r}(t)\right\rangle_{\mathrm{eq}} \times \\
\times \mathrm{e}^{i \mathbf{q r}} \mathrm{e}^{i \omega t} \mathrm{~d} t
\end{array}
$$

this expression is obtained by introducing at each site $r$ a $D$-dimensional vector $\mathbf{f}^{r}(\mathbf{Q})$ whose components $f_{l}^{r}$ are the different coherent form factors of the molecule $r$ in the orientations $l$, so that the actual molecular form factor is

$$
f^{r}(\mathbf{Q})=\sum_{l=1}^{D} \mu_{l}^{r} f_{l}^{r} .
$$

Define the scattering matrix $\boldsymbol{\Phi}(\mathbf{q}, \omega)$

$$
\Phi_{m l}(\mathbf{q}, \omega)=\int_{-\infty}^{+\infty} \Phi_{m l}(\mathbf{q}, t) \mathrm{e}^{-i \omega t} \mathrm{~d} t
$$

From $(21,22)$

$$
\chi_{l m}(\mathbf{q}, \omega)-\chi_{m l}(\mathbf{q}, \omega)^{*}=-i \omega \Phi_{l m}(\mathbf{q}, \omega)
$$

leading to a relationship between the $\Phi_{l m}$ 's and the susceptibilities.

If there is one molecule per unit cell we then get

$$
I_{\mathrm{c}}(\mathbf{Q}, \omega)=\operatorname{Tr}\left[\mathbf{F}_{(\mathbf{Q})}^{\mathrm{T}} \boldsymbol{\Phi}(\mathbf{q}, \omega)\right] \quad(\mathrm{T}=\text { Transposition })
$$

in which $\mathbf{F}(\mathbf{Q})=\mathbf{f} \otimes \mathbf{f}^{* \mathbf{T}}$ and $q=\mathbf{Q}-\mathbf{K}(\mathbf{K}$ : reciprocal lattice vector).
Going back to equation (24) we notice that when $l$ and $m$ are different $\Phi_{l m}$ and $\Phi_{m l}$ always occur together in the combination

$$
f_{l} f_{m}^{*} \Phi_{l m}(\mathbf{q}, \omega)+f_{m} f_{l}^{*} \Phi_{m l}(\mathbf{q}, \omega) .
$$

So that for an average centred structure we get

$$
I_{\mathrm{c}}(\mathbf{Q}, \omega)=\operatorname{Tr}\left[\mathbf{F}^{\mathrm{T}}\left(\frac{{\underline{\chi^{\prime \prime}}+\chi^{\prime \prime}}_{\omega}}{\omega}\right)\right]
$$

By integrating over energy the static susceptibility is obtained

$$
\begin{aligned}
\int_{-\infty}^{+\infty} \Phi_{m l}(\mathbf{q}, \omega) \mathrm{d} \omega=\Phi_{m l}(\mathbf{q}, t & =0)=\chi_{m l}(\mathbf{q})= \\
& =\chi_{m l}(\mathbf{q}, \omega=0) .
\end{aligned}
$$

Thus, the static scattering function reads

$$
I_{\mathrm{c}}(\mathbf{Q})=\operatorname{Tr}\left[\mathbf{F}^{\mathrm{T}}(\mathbf{Q}) \chi(\mathbf{q})\right] .
$$

3. Theoretical results and comparison with the experiments.

3.1 Static COHERENT SCATtERING. - M. More et al. have measured the static scattering intensity $I_{\mathrm{c}}(\mathbf{Q})$ in * $\mathrm{CBr}_{4}$ at different temperatures [10]. The measured intensities are very intense and anisotropic in the 220 zone appearing nearly as lines of strong scattering in the $[h \bar{h} \bar{h}]^{*}$ direction with maximum of intensity centered near $(2.05,2.05,0)$. The experimental intensity shows only a slight temperature response without any trace of critical scattering. The full width at half maximum (FWHM) of $I_{c}(\mathbf{Q})$ is $\Delta Q_{\perp}=0.28 \AA^{-1}$ in the [hh0]* direction and an anisotropy of $\Delta Q_{\|} / \Delta Q_{\perp}=4$ is found between FWHM parallel and perpendicular to the relevant $[h \hbar \hbar]^{*}$ direction.

If one sets $\lambda=1$ in (17) and so assumes that a strict all or nothing interaction describes the steric hindrance, the present theory (Eq. (31)) yields an intense cigar shaped $I_{c}(\mathbf{Q})$ scattering zone, similar to the experimental one but exactly centred at 220 where it diverges. Taking into account the softening $(\lambda<1)$, one finds that the scattering does not diverge and that the maximum slightly shifts towards higher Bragg angles showing an increasing influence of the molecular form factors. The features of the experimental scattering at $T=325 \mathrm{~K}$ are found for $\lambda=0.90$ 
corresponding to a mean repulsive energy of about $W=2.3 k T$. Precisely : the localization of the maximum $(2.05,2.05,0)$, the perpendicular FWHM of the cigar $\Delta Q_{\perp}=0.28 \AA^{-1}$ and the anisotropy $\Delta Q_{\|} / \Delta Q_{\perp} \sim$ 3.8. The results are presented in figure 5 . We have previously (2) shown the strong influence of the steric effects on the static scattering of $\mathrm{CBr}_{4}$ by use of a weak graph expansion method [2]. The present calculation confirms this fact and shows that the closed form approximation used here is reliable in the study of correlations induced by strong interactions.
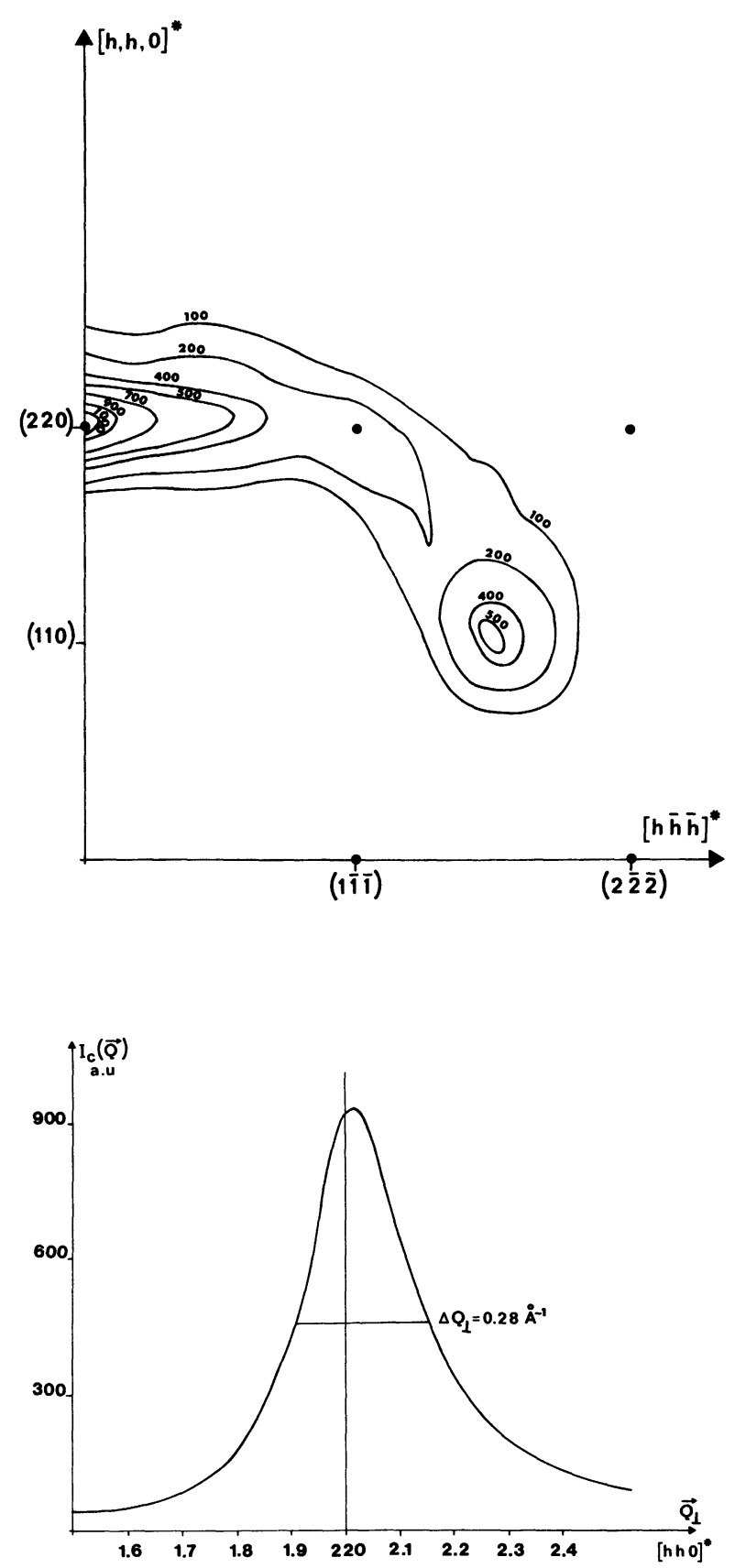

Fig. 5. - 5a theoretical isoscattering pattern in the $(11 \overline{2})^{*}$ plane showing an intense cigar shaped figure $(\lambda=0.90)$. $5 \mathrm{~b}$ static intensity in the $[h h 0]^{*}$ direction $(\lambda=0.90)$.
3.2 INELASTIC COHERENT SCATTERING. - Under the assumptions included in the model described in $\$ 2.1$ we have calculated the inelastic coherent scattering $I_{\mathrm{c}}(\mathbf{Q}, \omega)$ using the softening parameter $\lambda=0.90$ which correctly fits the experimental and theoretical static data. The results of the calculation at $T=325 \mathrm{~K}$ are summarized in figure 6. At each $Q$ point, the theoretical $I_{\mathrm{c}}(\mathbf{Q}, \omega)$ appears to be close to a Lorentzian. The absolute value of the line-width is not given by the theory since the height of the potential barrier between equilibrium orientations is not introduced; and the microscopic time scale, $\tau$, on which an uncorrelated reorientation takes place has been taken as unity in presenting the results. The calculation leads to important $\mathbf{Q}$ variations of the line-width in the static cigar zone. $\mathbf{Q}$ dispersion curves of the HWHM $\Gamma(\mathbf{Q})$ along the relevant $[h h 0]^{*}$ and $[h \bar{h} \bar{h}]^{*}$ directions are depicted in figure $6 b$. They display a strong dispersion along the short $[h h 0]^{*}$ axis whereas the dispersion is flat along $[h \bar{h} \bar{h}]^{*}$. An inelastic broadening of $\frac{\Gamma(2.5,2.5,0)}{\Gamma(2,2,0)} \sim 10$ is found in the direction where the static intensity falls down rapidly. The theoretical ratio of the $I_{\mathrm{c}}(\mathbf{Q}, \omega)$ maxima is :

$$
\frac{\mathrm{I}(2.15,2.15,0, \omega=0)}{\mathrm{I}(2.5,2.5,0, \omega=0)} \sim 20 .
$$

The $\mathbf{Q}$ dependence of the quasielastic component observed by More et al. [9, 10, 13] does show the features predicted by the present calculation. The inelastic broadening detected in the [hh0]* direction at $T=325 \mathrm{~K}$ is such that $\Gamma(2.1,2.1,0) \sim 0.03 \mathrm{THz}$ whereas $\Gamma(2.5,2.5,0) \sim 0.5 \mathrm{THz}$. The experimental ratio of the $I_{\mathrm{c}}(\mathbf{Q}, \omega)$ maxima is :

$$
\frac{\mathrm{S}(2.15,2.15,0, \omega=0)}{\mathrm{S}(2.5,2.5,0, \omega=0)} \sim 12 \text {. }
$$

Thus the calculated contribution of the steric restrictions to the dispersion rate of $\Gamma(\mathbf{Q})$ gives a correct quantitative interpretation of the experiments to within a factor of less than 2. No adjustable parameters are included except the softening parameter $\lambda$ which is fixed once and for all. More et al. have attributed the less intense and broader $(0.3 \mathrm{THz}<$ HWHM $<0.6 \mathrm{THz}$ ) inelastic scattering to some kind of " incoherent " component present in all the space and due to the monomolecular motion (self correlation). The present theory interprets the observed spectra as resulting from a continuous $q$ evolution due to the collective behaviour induced by the steric restrictions. A definitive conclusion needs more experimental information about the dispersion of $\Gamma(\mathbf{Q})$ especially between $2.1,2.1,0$ and $2.5,2.5,0$.

In figures $6 b$ the horizontal line at $\Gamma \simeq 6$ corresponds to the HWHM of an uncorrelated molecule (molecule only submitted to the thermal bath and the potential barriers with a relaxation time $\tau$ ). 

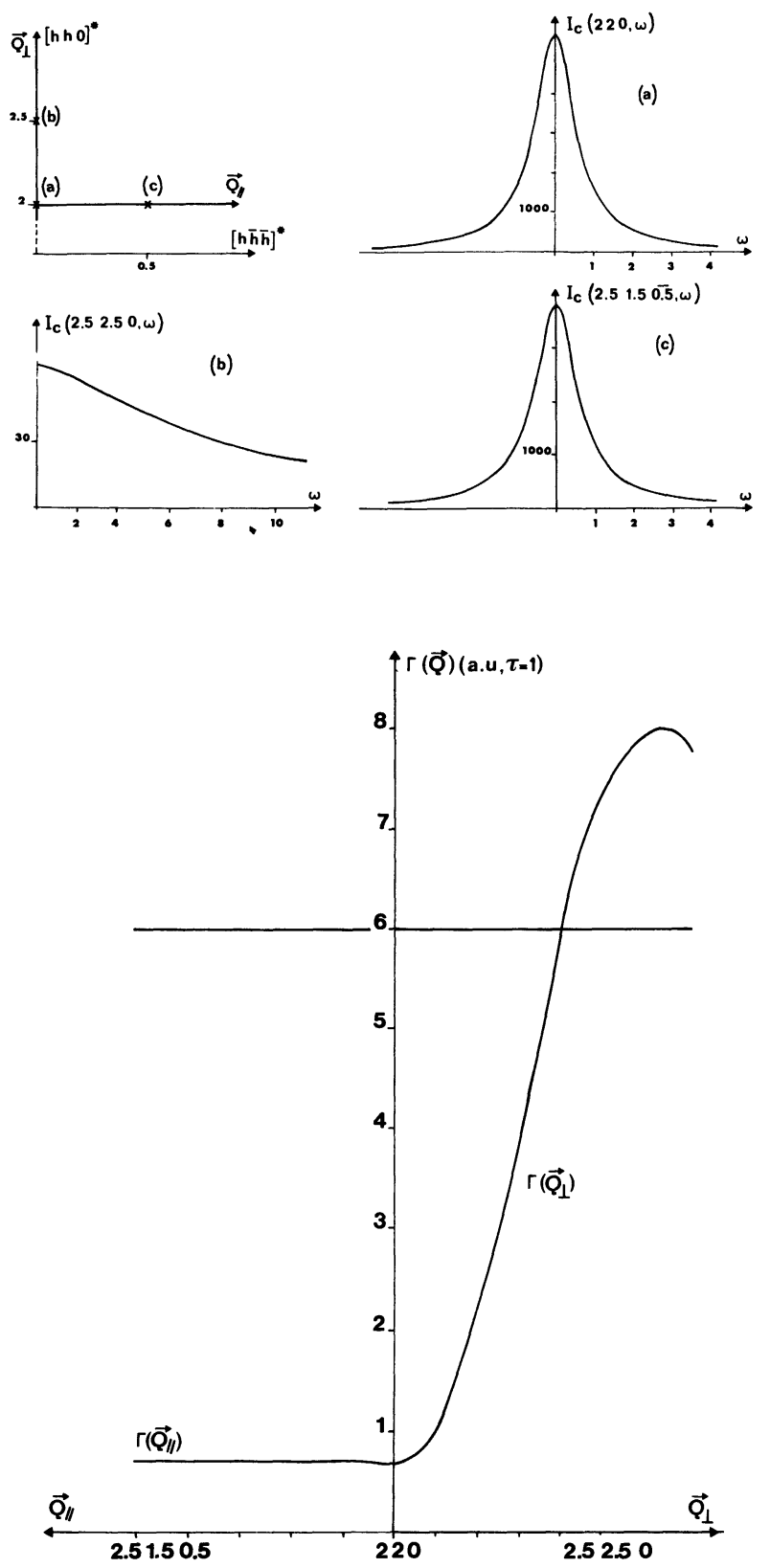

Fig. 6. - 6a typical constant $Q$ scans at the (a) (b) and (c) points $(\lambda=0.90) . \omega /$ in unity $\tau_{0}=1 ; 6 \mathrm{~b} Q$ dispersion curves of the HWHM $\Gamma(\mathbf{Q})$ in the $[h h 0]^{*}$ and $[h \overline{h \bar{h}}]^{*}$ directions. The horizontal line at $\Gamma=6$ corresponds to the HWHM of an uncorrelated molecule.

Whereas the maximum slowing down occurs in the strong static correlation zone (near 220) we can notice an " acceleration" for $h>2.4$. The value of the experimental width at 2.4,2.4, 0 could thus give an idea of $\tau$. At 2.5, 2.5, 0 , where the acceleration is not too important, it gives the lower limit

$$
\tau \simeq \frac{1}{2 \Pi \Gamma_{\text {exp }}} \simeq 0.3 \mathrm{pcs} \text {. }
$$

The (" coherent ") relaxation time $\tau_{\text {self }}$ of a single molecule experiencing reorientations correlated with its neighbours can be directly deduced from (13) and (16) in term of $\tau$ by eliminating the site labelling.

Identifying the following differential equation

$$
\begin{aligned}
\tau \mathrm{d} \frac{\left\langle\mu_{1}\right\rangle}{\mathrm{d} t}=- & 5\left\langle\mu_{1}\right\rangle\left(1-2 \lambda\left\langle\mu_{1}\right\rangle\right)^{4}+ \\
& +\left(1-2 \lambda\left\langle\mu_{1}\right\rangle\right)^{4}\left(\left\langle\mu_{2}\right\rangle+\left\langle\mu_{3}\right\rangle\right. \\
& \left.+\left\langle\mu_{4}\right\rangle+\left\langle\mu_{5}\right\rangle+\left\langle\mu_{6}\right\rangle\right)
\end{aligned}
$$

with the a priori rate equation for the (" coherent ") self molecular motion

$$
\begin{aligned}
\tau_{\text {self }} \mathrm{d} \frac{\left\langle\mu_{1}\right\rangle}{\mathrm{d} t}=-5\left\langle\mu_{1}\right\rangle+\left(\left\langle\mu_{2}\right\rangle+\left\langle\mu_{3}\right\rangle+\right. \\
\left.+\left\langle\mu_{4}\right\rangle+\left\langle\mu_{5}\right\rangle+\left\langle\mu_{6}\right\rangle\right) .
\end{aligned}
$$

We get

$$
\begin{gathered}
\tau_{\text {self }}=\frac{\tau}{(1-2 \lambda\langle\mu\rangle)^{4}} \simeq 4.16 \tau \simeq 1,3 \mathrm{pcs} \\
\left(\lambda=0.90 ;\langle\mu\rangle=\frac{1}{6}\right) .
\end{gathered}
$$

The present calculation only distinguishes the sterically different orientations of the $\mathrm{CBr}_{4}$ molecule and could be used for comparison with quadrupole spin lattice relaxation for example.

If the distinction between the sterically equivalent positions is introduced in the calculation, by labelling the $\mathrm{Br}$ atoms, we thus get a relaxation time which is measured in an experiment of the incoherent neutron type.

$\tau_{\text {self }}$ has thus more physical significance than $\tau$ and gives an idea of the global dynamic effect of the correlations.

$\tau$ and $\tau_{\text {self }}$ are relaxation times; they have thus the size of residence times $\left(\tau_{\mathrm{r}}\right)$. The value of the shorter jump time $\left(\tau_{\mathrm{j}}\right)$ whose duration has been neglected in the present theory will be estimated below.

Indeed a rapid discussion of the temperature evolution of the HWHM can give an insight into the validity of a jump model in the particular case of $\mathrm{CBr}_{4}$.

M. More [13] has measured the temperature evolution of $\Gamma(2.1,2.1,0)$ figure 7 . In the frame work of our model this evolution can be attributed to two factors :

- first the temperature evolution linked to the Boltzmann factor corresponding to the repulsive energy $W(18)$. This evolution, induced by the intermolecular correlations can be directly derived from the present calculation. Its contribution $\Delta \Gamma_{\mathrm{c}}(2.1,2.1,0)$ is given in figure 7 ;

- the second contribution is due to the temperature dependence of the non collective relaxation time $\tau$ which is taken to be given by an Arrhenius law

$$
\tau(T)=\tau_{0} \exp (A / k T)
$$




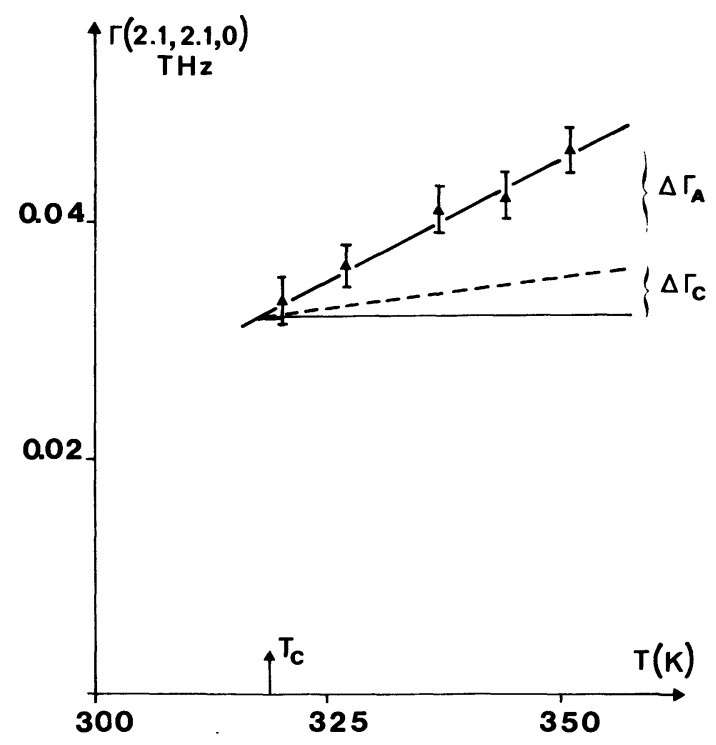

Fig. 7. - Temperature evolution of $\Gamma(2.1,2.1,0)$; experimental (M. More $(9,13))$; --.--.-- contribution of the correlations (present calculation). The difference gives the contribution of the activation energy $A$. with an activation energy $A$. This latter quantity is essentially given by the height of the potential barrier between the equilibrium orientations. The corresponding $\Delta \Gamma_{A}(2.1,2.1,0)$ contribution is given by the experimental $\Delta \Gamma(2.1,2.1,0)$ after subtraction of $\Delta \Gamma_{\mathrm{c}}$. It leads to an activation energy $A \approx 2300 \mathrm{cal} /$ mole $=3.6 k T$. The ratio $\tau_{\mathrm{j}} / \tau_{\mathrm{r}}$ of the average time during which a molecule jumps a barrier to the average time it resides in an equilibrium orientation can be approximated by $[18,19]$

$$
\frac{\tau_{\mathrm{j}}}{\tau_{\mathrm{r}}}=\frac{\exp (-A / k T)}{1-\exp (-A / k T)} \approx 0.028(\text { at } 320 \mathrm{~K})
$$

This result and the good agreement between experimental and calculated $S(\mathbf{Q}, \omega)$ spectra leads us to believe that $\mathrm{CBr}_{4}$ is still a case where an instantaneous jump model can be used in a first approximation; recognizing that its applicability is less reliable than in the prototype case of $\mathrm{NH}_{4} \mathrm{Cl}(A \approx 4220 \mathrm{cal} / \mathrm{mole}$ [8]) for example.

\section{Appendix.}

The complete set of consistency equations can be deduced from (19) by symmetry.

In terms of Fourier transform they read :

and

$$
[M] \boldsymbol{\delta}(\mathbf{q}, t)=[S] \boldsymbol{\beta}(\mathbf{q}) \mathrm{e}^{-i \omega t}
$$

$$
\boldsymbol{\delta}(\mathbf{q}, t)=\chi(\mathbf{q}, \omega) \boldsymbol{\beta}(\mathbf{q}) \mathrm{e}^{-i \omega t}
$$

We find the following value for $[M]$ in $\mathbf{q}(h, k, l)$

$$
\left[\begin{array}{cccccc}
1 & 1 & 1 & 1 & 1 & \\
\left(T_{1}-\frac{6}{2}-i \omega \tau\right) & \left(T_{2}-\frac{3}{2} \mathrm{e}^{i \Pi(h-k)}\right) & \left(T_{3}-\frac{3}{2} \mathrm{e}^{-i \Pi(h+l)}\right) & -2 T_{4} & \left(T_{5}-\frac{3}{2} \mathrm{e}^{i \Pi(h+k)}\right) & \left(T_{6}-\frac{3}{2} \mathrm{e}^{i \Pi(l-k)}\right) \\
\left(T_{1}-\frac{3}{2} \mathrm{e}^{i \Pi(k-h)}\right) & \left(T_{2}-\frac{6}{2}-i \omega \tau\right) & \left(T_{3}-\frac{3}{2} \mathrm{e}^{i \Pi(l-k)}\right) & \left(T_{4}-\frac{3}{2} \mathrm{e}^{i \Pi(h+k)}\right) & -2 T_{5} & \left(T_{6}-\frac{3}{2} \mathrm{e}^{-i \Pi(k+l)}\right) \\
\left(T_{1}-\frac{3}{2} \mathrm{e}^{i \Pi(h+l)}\right) & \left(T_{2}-\frac{3}{2} \mathrm{e}^{i \Pi(k-l)}\right) & \left(T_{3}-\frac{6}{2}-i \omega \tau\right) & \left(T_{4}-\frac{3}{2} \mathrm{e}^{i \Pi(l-h)}\right) & \left(T_{5}-\frac{3}{2} \mathrm{e}^{-i \Pi(k+l)}\right) & -2 T_{6} \\
-2 T_{1} & \left(T_{2}-\frac{3}{2} \mathrm{e}^{-i \Pi(h+k)}\right) & \left(T_{3}-\frac{3}{2} \mathrm{e}^{i \Pi(h-l)}\right) & \left(T_{4}-\frac{6}{2}-i \omega \tau\right) & \left(T_{5}-\frac{3}{2} \mathrm{e}^{i \Pi(k-h)}\right) & \left(T_{6}-\frac{3}{2} \mathrm{e}^{-i \Pi(h+l)}\right) \\
\left(T_{1}-\frac{3}{2} \mathrm{e}^{-i \Pi(h+k)}\right) & -2 T_{2} & \left(T_{3}-\frac{3}{2} \mathrm{e}^{i \Pi(k+l)}\right) & \left(T_{4}-\frac{3}{2} \mathrm{e}^{i \Pi(h-k)}\right) & \left(T_{5}-\frac{6}{2}-i \omega \tau\right) & \left(T_{6}-\frac{3}{2} \mathrm{e}^{i \Pi(-k)}\right)
\end{array}\right]
$$

where

$$
T_{1}=\exp (i \pi h) \cos \pi l+\exp (-i \pi h) \cos \pi k
$$

the five other $T_{i}$ factors are obtained by permutation of $\pm h, \pm k, \pm l$. The $S$ matrix has the following form

$$
[S]=\frac{1}{6}\left[\begin{array}{rrrrrr}
0 & 0 & 0 & 0 & 0 & 0 \\
-5 & 1 & 1 & 1 & 1 & 1 \\
1 & -5 & 1 & 1 & 1 & 1 \\
1 & 1 & -5 & 1 & 1 & 1 \\
1 & 1 & 1 & -5 & 1 & 1 \\
1 & 1 & 1 & 1 & -5 & 1
\end{array}\right]
$$




\section{Acknowledgments.}

The author wishes to thank M. More for a kind collaboration.

\section{References}

[1] Descamps, M., Coulon, G., Chem. Phys. 25 (1977) 117.

[2] Coulon, G., Descamps, M., J. Phys. C. 13 (1980) 2847

[3] Descamps, M., Coulon, G., J. Phys. C. 14 (1981) 2297.

[4] Huller, A., Press, W., Phys. Rev. Lett. 29 no 5 (1972) 266.

Press, W., Huller, A., Stiller, H., Stirling, W., Currat, R., Phys. Rev. Lett. 32 n 24 (1974) 1354.

[5] Descamps, M., J. Phys. C 15 (1982) 7265.

[6] Glauber, R. J., J. Math. Phys. 4, no 2 (1963) 294-307.

[7] Suzuki, M., Kubo, R., J. Phys. Soc. Japan. 24, no 1 (1968) 51-60.

[8] Michel, K. H., J. Chem. Phys. 58, no 1 (1973) 142-152.

[9] More, M., thèse d'Etat (1982) Lille.
[10] More, M., Lefebvre, J., Hennion, B., Powell, B. M., Zeyen, C. M. E., J. Phys. C. 13 (1980) 2833-46.

[11] Nagle, J. F., J. Math. Phys. 9 (1968) 1007.

[12] More, M., Lefebvre, J., Fouret, R., Acta Cryst. B33 (1977) 3862.

[13] More, M., Lefebvre, J., Hennion, B., J. Physique 45 (1984).

[14] Kubo, R., J. Phys. Soc. Japan 12 (1957) 570.

[15] Van Hove, L., Phys. Rev. 95, no 1 (1954) 249.

[16] Marshall, W., Lovesey, S. W., Theory of thermal Neutron Scattering (Oxford University Press) 1971.

[17] Pick, R. M., Yvinec, M., J. Physique 41 (1980) 1053.

[18] De Micheli, R., Guilotto, L., Nardelli, G. F., Phys. Rev. 131, no 4 (1963) 1619.

[19] Fontaine, D., thèse d'Etat, Paris (1978) p. 78-80. 